\title{
Black Ginseng
}

Created by: De Qiang Dou

Version received: 24 May 2019

check for updates

Black ginseng is a processed ginseng which prepared by steaming and drying of white or red ginseng for several times (usually 9). This process resulting in extensive changes in types and amounts of several secondary metabolites. Thus, primary ginsenosides (the main active inredients in ginseng) were transformed into less polar derivatives by steaming. In addition, apparent changes happened to other secondary metabolites such as the increasing of phenolic compounds, reducing sugars and acidic polysaccharides as well as the decrease in concentrations of free amino acids and total polysaccharides. Furthermore, the presence of some Maillard reaction products like maltol was also engaged. These obvious chemical changes were associated with a noticeable superiority for black ginseng over white and red ginseng in most of the comparative biological studies including anticarcinogenic, immunomodulatory, anti-Inflammatory, antidiabetic, hepatoprotective, antioxidant and tonic effects.

\section{Introduction}

Ginseng is the roots and rhizomes of Panax ginseng Mey., belonging to the perennial plants of genus Panax and family Araliaceae. The most commonly used species are: Panax ginseng (Asian ginseng), Panax quinquefolius (American ginseng) and Panax notoginseng (Chinese notoginseng or Sanqi) 1 . Ginseng has been used in China for more than 4000 years as a tonic and restorative, promoting health, treating hemorrhage, impotence, anorexia and infections. Modern clinical studies proved various pharmacological effects for ginseng. For instance, the aphrodisiac and adaptogenic properties of ginseng were reported to result from its effects on the hypothalamic-pituitary-adrenal axis, resulting in an elevation of corticotropin and corticosteroids levels in plasma[2]. Moreover, the immune enhancing activity was correlated to its ability to regulate different types of immune cells such as dendritic cells, macrophages, natural killer cells, B cells and T cells [3]. Induction of apoptosis, inhibition of angiogenesis of cancer cells in addition to inhibition of cell proliferation and immunosurveillance are reported mechanisms for the anticancer activities of Panax ginseng [4]. According to in vitro and in vivo results, ginseng could treat cardiovascular diseases through antioxidation properties, reduction of platelet adhesion, vasomotor regulation, enhancing lipid profiles, and influencing several ion channels [5]. What is more, ginseng exhibited treating effects in many central nervous system disorders such as Parkinson's disease, Alzheimer's disease, depression, cerebral ischemia as well as several other neurodevelopmental disorders. Ginseng could exert these activities through different pathways such as neuroprotection, synaptic plasticity regulation, decreasing neuroinflammatory processes and regulation of neurotransmitter release [6].

The main bioactive secondary metabolites in ginseng are ginsenosides, which belong to dammarane-type triterpene saponins with different sugar moieties attached at C-3 and C-20. Ginsenosides are named ' $R x^{\prime}$ ': 'R' refers to the root and ' $x$ ' describes the ascending alphabetical order of their chromatographic polarity. Accordingly, Ra is the highest polar ginsenoside, while Rb is less polar than Ra [7]. As shown in Figure 1, ginsenosides can be classified into two major classes; (1) the protopanaxadiol (PPD) type in which the sugar units are attached to the $\beta-\mathrm{OH}$ at C-3 and/or C-20 (such as $R a_{1}, R a_{2}, R a_{3}, R b_{1}, R b_{2}, R_{c}, R_{d}, R g_{3}, R h_{2}$ and $R_{1}$ ) and (2) the protopanaxatriol (PPT) type in which the sugar units are attached to the $\alpha-O H$ at $C-6$ and/or the $\beta-\mathrm{OH}$ at C-20 (such as Re, Rf, $\mathrm{Rg}, \mathrm{Rg}_{1}, \mathrm{Rg}_{2}$ and $\mathrm{Rh}_{1}$ ). Moreover, some other ginsenosides, such as ocotillol group, have a five-membered epoxy ring at C-20 (for instance F11) and the oleanolic acid saponins (for example, Ro, C-IV and C-lb) have also been isolated and identified [의. The major ginsenosides in the white or (sun-dried) ginseng's roots are $R g_{1}, R e, R b_{1}, R c, R b_{2}, R b_{3}$ and $R d$, which make up more than $70 \%$ of total ginsenosides [9]. In addition, some PPD or PPT-type ginsenosides with structure changes in side-chain were isolated and exhibited strong biological activities [10].

Figure 1. Major classes of Ginsenoside.

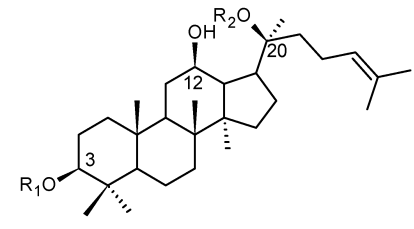

Protopanaxadiol (PPD) ginsenosides

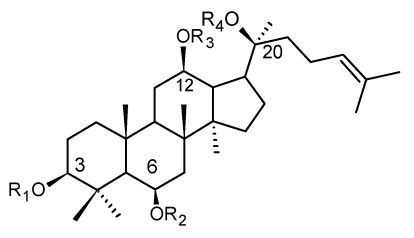

Protopanaxatriol (PPT) ginsenosides

According to our previous research, the three generally-used species in Panax genus could be distinguished by the presence of some characteristic ginsenosides such as the notoginsenoside $R_{1}$, which is a characteristic marker for $P$. notoginseng, while the ginsenoside Rf is a marker for $P$. ginseng. Furthermore, the ocotillol-type triterpene 24(R)-pseudo-ginsenoside $\mathrm{F}_{11}$ presents in high amounts in $P$. quinquefolius and in very minute amounts in $P$. ginseng, and hence a high ginsenoside Rf/24(R)-pseudo-ginsenoside $\mathrm{F}_{11}$ ratio $(>700)$ clearly differentiates $P$. ginseng and $P$. quinquefolius [11]. Likewise, the ratio between $\mathrm{Rb}_{1}$ and $\mathrm{Rg} \mathrm{g}_{1}$ is a very clear marker as the high $\mathrm{Rb} \mathrm{b}_{1} / \mathrm{Rg}_{1}$ ratio (around 10 or greater) indicates $P$. quinquefolius, while low (1-3) indicates $P$. ginseng [12]. Additionally, the ginseng plant contains some other important secondary metabolites such as ginseng oils, phytosterol, carbohydrates, amino acids, peptides, vitamins, minerals, certain enzymes and phenolic compounds (caffeic acid, syringic acid, p-coumaric acid, ferulic acid and cinnamic acid) [13].

Due to black ginseng having originated and being distributed in Asia, the term black ginseng in this article refers to black $P$. ginsing, unless otherwise stated. 


\section{Phytochemical changes}

The preparation of balck ginseng depends on the steaming and drying for several times (usually 9). The steaming process casues the transformation of several secondary metabolites in to other forms through different chemical reactions. The polar ginsenosides transform into specific less polar ginsenosides by hydrolysis, dehydration, decarboxylation and isomerization reactions. The notable structural changes are the hydrolysis of sugar moieties at C-3, C-6 or C-20 and subsequent dehydration at C-20. These reactions took place to the major primary ginsenosides (Rb 1 , Rb 2 , Rc, Rd, Re

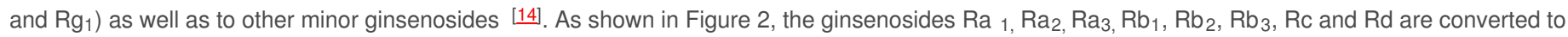
the ginsenosides $\mathrm{Rg}_{3}, \mathrm{~F}_{2}$, compound $\mathrm{K}$ and $\mathrm{Rh} 2$ in black ginseng through hydrolysis reactions for sugar moieties at $\mathrm{C}-3$ and $\mathrm{C}-20$. On the other side, the ginsenosides $\mathrm{Rk}_{1}$ and $\mathrm{Rg}_{5}$ are produced as a result of the dehydration reactions of $\mathrm{Rg}{ }_{3}$ [15] . The $\mathrm{R}$-epimers of $\mathrm{Rg} 2$, $\mathrm{Rg} \mathrm{g}_{3}$ and $\mathrm{Rh} \mathrm{H}_{1}$ were produced through isomerization of the corresponding ginsenosides and through addition reactions (selective attack of the hydroxyl group) for the dehydrated corresponding ginsenosides $\left(\mathrm{Rk}_{1}\right.$ and $\mathrm{Rg}_{5}$ in case of $\left.20(\mathrm{R})-\mathrm{Rg} \mathrm{g}_{3}\right)$ [16].

$\mathrm{Rh}_{1}$ and $\mathrm{Rh}_{4}$ were deduced to be generated from the ginsenoside $\mathrm{Rg}_{1}$ through hydrolysis and dehydration reactions, respectively. Interestingly, four ginsenoside $\left(\mathrm{Rg}_{6}, \mathrm{~F}_{4}, \mathrm{Rk}_{3}\right.$, and $\left.\mathrm{Rh}_{4}\right)$ were produced from the ginsenoside $\mathrm{Rg}_{2}$ through hydrolysis and dehydration reactions [17]. The acetylated ginsenosides (20(S)-Rs 3 and $20(R)-R s_{3}$ ) were produced from the malonyl derivatives of $R b_{1}, R b_{2}$, Rc and Rd the by hydrolysis of glycosyl moiety at C20 and decarboxylation of malonyl moiety attached to glycosyl linkage at C-3, 20(S)-Rs $s_{3}$ further underwent dehydration to generate Rs 4 and Rs . Generally, The steaming process causes an increase in the protopanaxadiol group to protopanaxatriol group ratio (PD/PT) from 1.9 to 8.4 in white and black ginseng, respectively [15] in addition, it also led to the production of some specific ginsenosides such as (20(S)-, 20(R)- $\mathrm{Rg}_{3}, \mathrm{Rk}_{3}, \mathrm{Rh}_{4}, \mathrm{Rk}_{1}, \mathrm{Rg}_{5}$, ...etc.) which are absent from white ginseng. As black ginseng was subjected to much more steaming, the concentration of these ginsenosides is much higher than red ginseng. Similarly, the steaming and drying processes led to some chemical changes in the other secondary metabolites. Although steaming causes a decrease in the contents of polysaccharides from $29.1 \%$ in fresh ginseng to be only $11.1 \%$ in black ginseng [18], it causes an increase in reducing sugars and acidic polysaccharides contents. The increase of reducing sugars and acidic polysaccharides contents in black ginseng with percentages of $128 \%$ and $187.5 \%$, respectively, comparing the white ginseng was reported [16]. Similarly, the phenolic compounds content was increased more than three folds by steaming from $3.1 \mathrm{mg} / \mathrm{g}$ in white ginseng to be $10.6 \mathrm{mg} / \mathrm{g}$ in black ginseng $[16]$. In another comparative study, the phenolic contents of white ginseng and black ginseng roots of Panax ginseng, $P$. notoginseng and $P$. quinquefolium were evaluated to be $20.4 \pm 0.90 \mathrm{mg} / \mathrm{g}, 17.12 \pm 0.56 \mathrm{mg} / \mathrm{g}, 14.45 \pm 0.13 \mathrm{mg} / \mathrm{g}$ in white ginseng roots and to $34.3 \pm 0.18,44.15 \pm 1.45$, and $34.05 \pm 2.03 \mathrm{mg} / \mathrm{g}$ in the black ginseng roots, respectively. The identified phenolics included; ferulic, gentisic, cinnamic, syringic, and p-hydroxybenzoic acids combined with arginine and maltose due to Maillard reaction [19]. Another study reported the increase of salicylic acid, vanillic acid and p-coumaric acid contents from $0.121,0.404$ and $0.522 \mathrm{mg} / 100 \mathrm{~g}$ in white ginseng to $0.394,0.628$ and $0.737 \mathrm{mg} / 100 \mathrm{~g}$ in black ginseng, respectively, because of the steaming process [ $\underline{20}$. In fact, the black color of black ginseng is a result of a chemical reaction named Maillard reaction, which is achemical reaction between reducing sugars and amino acids resulting in glycosylamines and or ketosamines [21]. The reported Maillard reaction products in ginseng due to steaming included: Arginmaltose [20] Arg-fru-glc, Arg-fru, maltol-3-O- $\beta$-D-glucoside in addition to maltol which increased from $2.598 \mathrm{mg} / 100 \mathrm{~g}$ in white ginseng to 94.007 $\mathrm{mg} / 100 \mathrm{~g}$ by steaming [19]. The content of maltol was reported to be much higher in black ginseng than red and white ginseng [22]. The significant decrease of free amino acids from $17.9 \mathrm{mg} / \mathrm{g}$ in white ginseng to $2.79 \mathrm{mg} / \mathrm{g}$ after steaming has been reported [23]. Arginine's content (the most predominant amino acid in ginseng) was reduced from 10.4 to $1.38 \mathrm{mg} / \mathrm{g}$ and b-N-oxalyl-L-a,b-diaminopropionic acid (b-ODAP), a famous neurotoxin, was decreased also by $92.9 \%$. The decrease of amino acid content in black ginseng is believed to be a result of Maillard reaction due to the detection of increased levels of Maillard reaction products [22]. These results have been authenticated by a recent study used multiple ultra-performance liquid chromatography with mass spectrometry (UPLC-MS) assay methods and proved the decrease of amino acids contents during the steaming process. The concentrations of 29 amino acids were less in red ginseng than white ginseng and were the least in black ginseng [24]. On the other hand, benzo(a)pyrene hydrocarbon was detected only in the black ginseng with a content of $0.17 \mu \mathrm{g} / \mathrm{kg}[16]$. Benzo(a)pyrene is a polycyclic aromatic hydrocarbon produced as a byproduct of the incomplete burning of organic materials. It is a potent carcinogenic and listed as Group 1 carcinogens by the International Agency for Research on Cancer [24]. According to the Korea Food and Drug Administration (KFDA), the maximum acceptable level for benzo(a)pyrene on any food products is $5.0 \mu \mathrm{g} / \mathrm{kg}[16]$. Accordingly, although reporting the presence of benzo(a)pyrene hydrocarbon in black ginseng, it's still in very minute amounts and much lower than the accepted limit.

Figure 2. Pathways of chemical changes of PPD- type ginsenosides during black ginseng processing . 

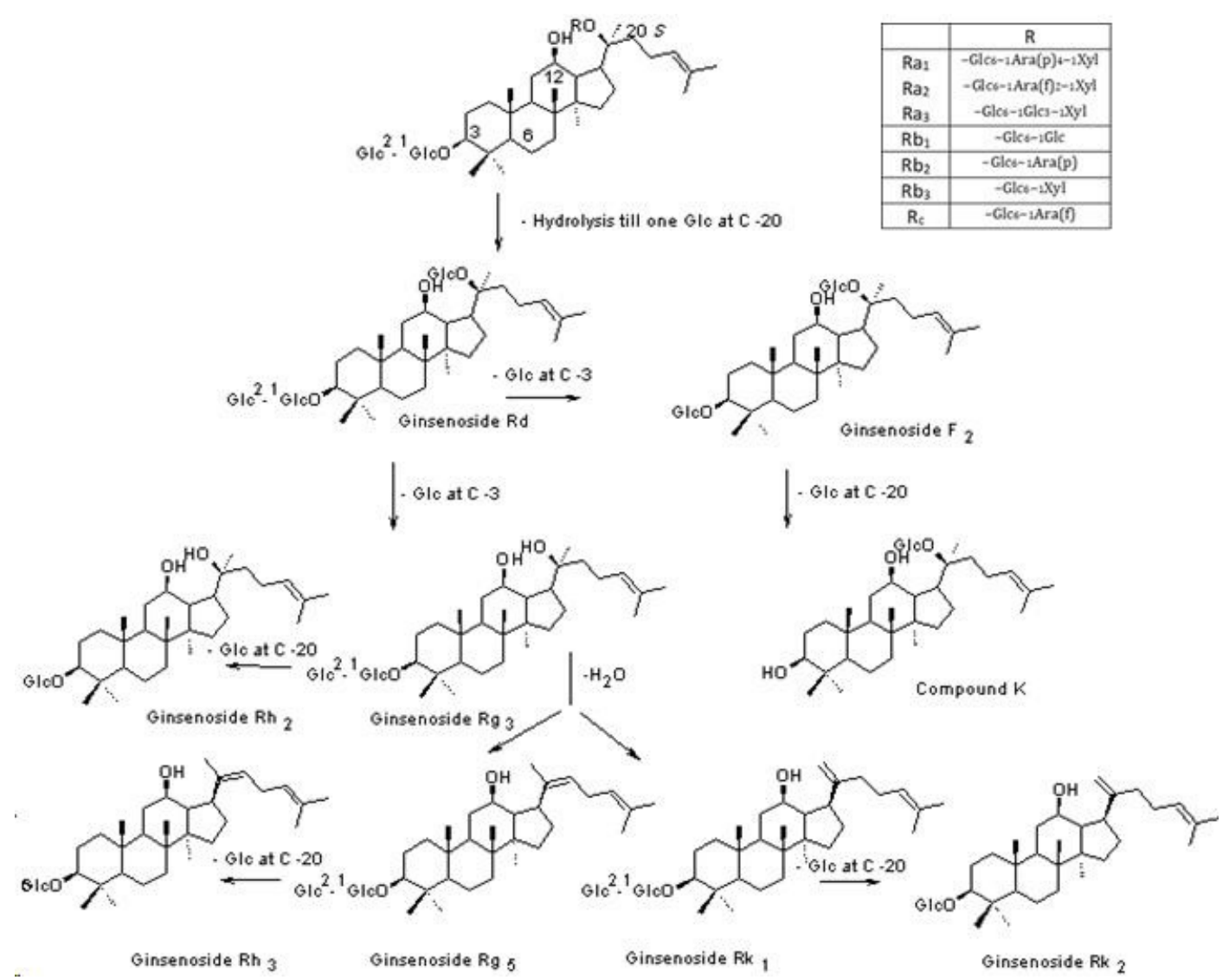

3. Pharmacological Studies

During the past decade, there was considerable interest in investigating black ginseng's pharmacological actions using different biochemical, pharmacological and molecular biological techniques. The examined pharmacological activities included anticancer, hepatoprotective, antidiabetic, antioxidant and general tonic activities besides its effect on the central nervous and immune systems. Some of these reports were carried out to compare the biological activity of black ginseng with white and/or red ginseng. While most of these reports were designed to figure out the pharmacological potential of black ginseng in addition to its mechanism of action. Furthermore, there is extensive literature involving the biological activities of the major transformed ginsenosides, such as $\mathbf{R g}_{3}, \mathbf{R k}_{3}, \mathbf{R h}_{2}, \mathbf{R k}_{1}$ and $\mathbf{R g}_{5}$, which are present in black ginseng in much higher concentrations than red ginseng and are totally absent in white ginseng. Additionally, the amounts of polyphenols, acidic polysaccharides and Maillard reaction products increase considerably by the steaming process to be in the highest concentrations in black ginseng.

\subsection{Anticarcinogenic Effects}

Black ginseng exerted promising in vitro and in vivo anticarcinogenic effects against several cancer types through different mechanisms of action. Black ginseng's crude saponin fraction exerted stronger in vitro cytotoxic activities than red ginseng against ACFIN, HCT-15 and PC-3 cell lines with $I_{50}$ values ranging from $60.3-90.8 \mu \mathrm{g} / \mathrm{mL}[25]$. In another comparative study, black ginseng extract exhibited cytotoxic activity against colon 26-M3.1 carcinoma cell line with an IC $_{50}$ value of $800 \mu \mathrm{g} / \mathrm{mL}$, while it was $2000 \mu \mathrm{g} / \mathrm{mL}$ for white ginseng[26]. In addition, 
black ginseng extract could inhibit basic fibroblast growth factor (bFGF)-induced endothelial cell proliferation and migration dosedependently through its ability to inhibit the angiogenesis process [27]. Furthermore, breast cancer MCF-7 cell lines proliferation was inhibited by black ginseng due to cell cycle arrest induction in the G0/G1 phase in a dose-dependent manner [28].

Black ginseng exhibited promising anticancer activities in different in vivo studies such as tumor inhibition in $\mathrm{H}_{22}$ tumor-bearing mice dosedependently via immune function improvement and tumor cell apoptosis induction [29]. Moreover, it performed in vivo anticancer activities against hepatocellular carcinoma as it reduced the size and the volume of an HepG2 cell transplanted tumor in BALB/c nude mice [30].

\subsection{Immunomodulatory and Anti-Inflammatory Effects}

In a comparative study, black ginseng exhibited stronger antiinflammatory and anti-nociceptive effects than red ginseng in xyleneinduced ear edema model in mice and carrageenan-induced paw edema in rats; it inhibited the pro-inflammatory mediators, inducible nitric oxide synthase (iNOS) and cyclooxygenase-2 (COX-2) and proinflammatory cytokines, IL-1 $\beta$, interleukin-6 (IL-6) and tumor necrosis factor- $\alpha$ (TNF- $\alpha$ ) [31]. In addition, the lipopolysaccharide-induced TNF$\alpha$ release was significantly decreased after treatment with black ginseng extract $[29]$. Furthermore, the dose-dependent recovering effect of black ginseng extract against the cisplatin-induced nephrotoxicity and the reduced (pig cell LLC-PK1) cells viability, has been indicated [32]. In addition, in the same experiment, compound $K$ could abrogate the elevated percentage of apoptotic LLC-PK1 cells significantly.

The black ginseng extract potently inhibited atopic dermatitis and asthma through suppression of the elevated IL-6 and IL-8 induced b y Dermatophagoides pteronissinus treatment in human acute monocytic leukemia (THP-1) and human eosinophilic leukemic (EoL1) cell lines [33]. Similarly, gamma-irradiated black ginseng extract could inhibit mast cell degranulation and suppress atopic dermatitislike skin lesions in mice through different mechanisms, including the suppression of $\beta$-hexosaminidase and histamine in the stimulated mucosal mast cells [34].

\subsection{Hepatoprotective Effect}

Black ginseng exhibited a hepatoprotective effect on acetaminopheninduced mice liver injury decreasing the levels of serum alanine aminotransferase (ALT), aspartate transaminase (AST) and lipid peroxidation product malondialdehyde (MDA) significantly. Meanwhile, the antioxidant levels in liver tissues were elevated, including glutathione (GSH), cytochrome P450 E1 (CYP2E1) and 4hydroxynonenal (4-HNE) [35]. Additionally, treatment with black 
ginseng ethanol extract decreased lipid accumulation in the liver and damage in the muscle of diabetic mice through the activation of AMPactivated protein kinase (AMPK) [36]. Symmetrically, black ginseng could decrease the activation of apoptotic pathways in the liver through decreasing Bax and increasing Bcl-2 protein expression levels associating a significant inhibition of APAP-induced necrosis and inflammatory infiltration [34]. Fermented black ginseng exhibited hepatoprotective activities against hydrogen peroxide $\left(\mathrm{H}_{2} \mathrm{O}_{2}\right)-$ mediated oxidative stress in HepG2 human hepatocellular carcinoma cell lines. It could attenuate the increased ROS level and elevate the expression and activity of antioxidant enzymes, such as superoxide dismutase, catalase and glutathione peroxidase. At the same time, black ginseng inhibited the phosphorylation of upstream mitogenactivated protein kinases (MAPKs) [37].

\subsection{Antidiabetic Effect}

In a comparative experiment, black ginseng ethanolic extract exerted stronger antidiabetic activity than red ginseng. It reduced hyperglycemia at a dose of $200 \mathrm{mg} / \mathrm{kg}$ in STZ-treated mice and improved the $\boldsymbol{\beta}$-cell function by inhibition of $\boldsymbol{\beta}$-cell apoptosis through suppression of the cytokine-induced nuclear factor-KB signaling pathway in the pancreas [38]. Black ginseng extract decreased the elevated blood glucose levels in streptozotocin-induced diabetic rats to a normal level [39]. Additionally, black ginseng ethanol extract decreased fasting blood sugar and glycated hemoglobin (HbA1c) via the upregulation of GLUT2 and GLUT4 expression in diabetic rats [35]. In another experiment, black ginseng ethanolic extract could significantly improve fasting blood glucose levels, glucose tolerance and decrease HbA1c in STZ-induced diabetic mice. It could increase glucose uptake in $\mathrm{C} 2 \mathrm{C} 12$ myotubes via AMPK, Sirt1 and PI3-K pathways. In addition, a significant increase in the expression of the genes involved in glucose uptake in the muscle (glucose transporter (GLUT)1, GLUT4) and $\beta$-oxidation (acyl-CoA oxidase (ACO), carnitine palmitoyl transferase 1a (CPT1a), mitochondrial medium-chain acylCoA dehydrogenase (MCAD)) has been reported [40].

\subsection{Anti-Obesity and Antihyperlipidemic Effects}

Black ginseng ethanol extract could decrease the induced hyperlipidemia and fat accumulation in white adipose tissues and liver in the fat diet-mice through the inhibition of fat digestion [41]. Black ginseng extract efficiently reduced the total serum cholesterol levels and low-density lipoprotein (LDL) levels in the fat diet-fed mice [42] and in STZ-induced diabetic mice [39]. In addition, a significant decrease in triglyceride and non-esterified fatty acid (NEFA) levels with an increase in HDL level was noticed in male obese diabetic C57BLKS/J-db/db mice after treatment with black 
ginseng [43]. Water and ethanol extracts of black ginseng decreased lipid accumulation through the regulation of PPARY, C/EBP $\alpha$ and AMPK phosphorylation in 3T3-L1 cells with a stronger activity for the ethanol extract. Black ginseng could regulate the expression of hepatic genes involved in gluconeogenesis (phosphoenol pyruvate carboxykinase (PEPCK), glucose6 phosphatase (G6Pase)), glycogenolysis (liver glycogen phosphorylase (LGP)) and glycogenesis (glycogen synthase (GS)) [39] and attenuate the key genes responsible for lipogenesis (acetyl-coenzyme $A$ (CoA) acetyltransferase, 2, 3-hydroxy-3-methyl-glutaryl-CoA reductase) [41].

3.6. Effects on the Central Nervous System

Black ginseng extract could prevent the cognitive impairment induced by cholinergic dysfunction through the inhibition of acetylcholinesterase (AChE) activity after $24 \mathrm{~h}$ of a single administration of $200 \mathrm{mg} / \mathrm{kg}$ in the brain [45]. In a comparative experiment, both white ginseng and black ginseng of the roots o f Panax ginseng, $P$. quinquefolium a nd $P$. notoginseng inhibited AChE and butyrylcholinesterase (BChE) dose-dependently. The efficacy of black ginseng roots was greater than that of the respective white ginseng roots of each species [46]. Black ginseng extract significantly reversed scopolamine (SCOP)-induced memory impairment in amnesic mice and also reduced escape latency by decreasing malondialdehyde (MDA) levels and restored superoxide dismutase (SOD) and catalase (CAT) activities [47]. The same effect was confirmed by the black ginseng-enriched formula (ChongMyung-Tang) in the water maze associating the inhibition of nitric oxide production in BV2 cells and significant suppression of expression of proinflammatory cytokines such as nitric oxide synthase, cyclooxygenase-2 and interleukin-1b. Over and above, the black ginseng-enriched CMT extract diminished the protein expression of MAP kinase and NF-kB pathway factors [48].

Likewise, black ginseng protected rats against ischemia-induced neuronal and cognitive impairment and improved the escape latency with reduced loss of cholinergic immunoreactivity and nicotinamide adenine dinucleotide phosphate-diaphorase

(NADPH-d)-positive neurons in the hippocampus [49]. After oral administration of black ginseng extract with a dose of $200 \mathrm{mg} / \mathrm{kg}$ for 16 weeks, the cognitive deficits associated with normal aging in old-age mice was decreased, associating DNA damage protection and a significant increase in brain-derived neurotrophic factor protein expression [50].

\subsection{Antioxidant Effect}

There was an increase in the content of total phenolics because of steaming. This increase is thought to be a direct reason for the higher antioxidant activity of black ginseng [51]. Black ginseng roots 
of Panax ginseng, $P$. quinquefolium and $P$. notoginseng exhibited more total phenolic contents and 2,2-diphenyl-1-picryl-hydrazyl (DPPH) scavenging activity than white corresponding roots. The best antioxidant activity was obtained with black ginseng roots of $P$. ginseng [45]. The same result was confirmed by another experiment through improving antioxidant activity according to the increasing number of steaming-drying cycles [52]. Black ginseng improved most of the morphological scores significantly in ethanol-treated embryos associating the significant restoration of the decreased mRNA levels of the antioxidant enzymes; cytosolic glutathione peroxidase (GPx), phospholipid hydroperoxide GPx and selenoprotein $P$, suggesting that black ginseng exerted this protective effect via the augmentation of antioxidative effect in the embryo [53]. Unexpectedly, in an acetaminophen-induced oxidative stress rat model, it was found that red ginseng extract exhibited more anti-oxidant activity than white ginseng and black ginseng extracts [54]. Four acidic polysaccharide fractions (BGP-60, BGP-65, BGP-70 and BGP-80) with estimated molecular weights of 28.6, 26.7, 11.4 and $3.05 \mathrm{kDa}$, respectively, were purified from black ginseng and showed strong potential antioxidant activities against DPPH, superoxide anion radicals and hydroxyl radical [55].

3.8. Tonic Effect

Black ginseng treatments at a dose of $150 \mathrm{mg} / \mathrm{kg}$ significantly increased the exercise capacity in rats. The level of blood lactic acid was decreased but the activity of citrate synthase in muscles was increased [56]. Black ginseng also increased muscle growth and could treat or prevent muscle loss related to aging through the production of myoblasts with larger multinucleated myotubes and increased diameter and thickness; the mechanism of action is thought to be the activation of Akt/mTOR/p70S6k axis [57]. The complex extract of black ginseng and fenugreek could increase cell viability, which had been attenuated because of oxidative stress through regulation of Erk kinase activation. Moreover, the oral administration of the complex could significantly increase the levels of total and bioavailable testosterone, follicle-stimulating hormone and luteinizing hormone in a hormone-deficient animal model. A dosage of $100 \mathrm{mg} / \mathrm{kg}$ of the complex extract could improve motor function and increase muscle endurance in a forced swimming test [58]. In a comparative study, the acidic polysaccharide of black ginseng exhibited a stronger antifatigue activity, than the neutral polysaccharide using the forced swim test through the restoring of the physiological markers for fatigue including glucose, glutathione peroxidase (GPx), creatine phosphokinase (CK), lactic dehydrogenase malondialdehyde (MDA) levels [59].

(LDH) and 3.9. Topical Uses 
Fermented black ginseng exhibited a significant anti-wrinkle effect at a concentration of $0.3 \mu \mathrm{g} / \mathrm{mL}$ through the increase of the type I procollagen expression levels in the human fibroblasts and the decrease in the MMP-1 expression level. Furthermore, at $3 \mu \mathrm{g} / \mathrm{mL}$ it increased the expression of TIMP-2 up to $154.55 \%$. However, at 10 $\mu \mathrm{g} / \mathrm{mL}$ it decreased the expression levels of MMP-2 and MMP-9 to $45.15 \%$ and $66.65 \%$, respectively $[60]$.

The wound healing activity of fermented black ginseng in human umbilical vein endothelial cells was mediated by angiogenesis through the MAP kinase pathway and enhanced the tube formation in HUVECs and migration in HaCaT cells was discussed [61]. Fermented black ginseng could stimulate the phosphorylation of p38 and extracellular signal-regulated kinase in HaCaT cells. Moreover, mice treated with $25 \mathrm{mg} / \mathrm{mL}$ exhibited faster wound closure in the experimental cutaneous wounds model [60].

3.10. Toxicity Studies

Single acute oral toxicity of black ginseng has been studied in rats indicating that the oral $L_{50}$ in the rats is higher than $15 \mathrm{~g} / \mathrm{kg}$, meaning that the black ginseng is virtually nontoxic [62]. On a cellular level, black ginseng showed no significant cytotoxicity against the normal splenocyte cells [27], while $R_{G_{3}}$ protected normal cells against cancer by reduction of MNNG-induced DNA damage and apoptosis [64]. The eye irritation potential of fermented black ginseng was examined using the EpiOcular-EIT kit; results indicated that black ginseng considered is safe for the eyes at concentrations up to 100 $\mu \mathrm{g} / \mathrm{m} \mathrm{L}[\underline{59]}$. Fermented black ginseng could ameliorate the nephrotoxicity via regulating oxidative stress, inflammation and apoptosis with a DPPH radical scavenging activity stronger than that of white ginseng. Moreover, the reduced creatinine clearance levels and cell viability by cisplatin were recovered significantly after treatment [63]. Similarly, black ginseng extract and $\mathrm{Rg}_{3}$ could recover the cisplatin-induced nephrotoxicity and the reduced (pig cell LLCPK1)cells yiability dose-dependently [31].

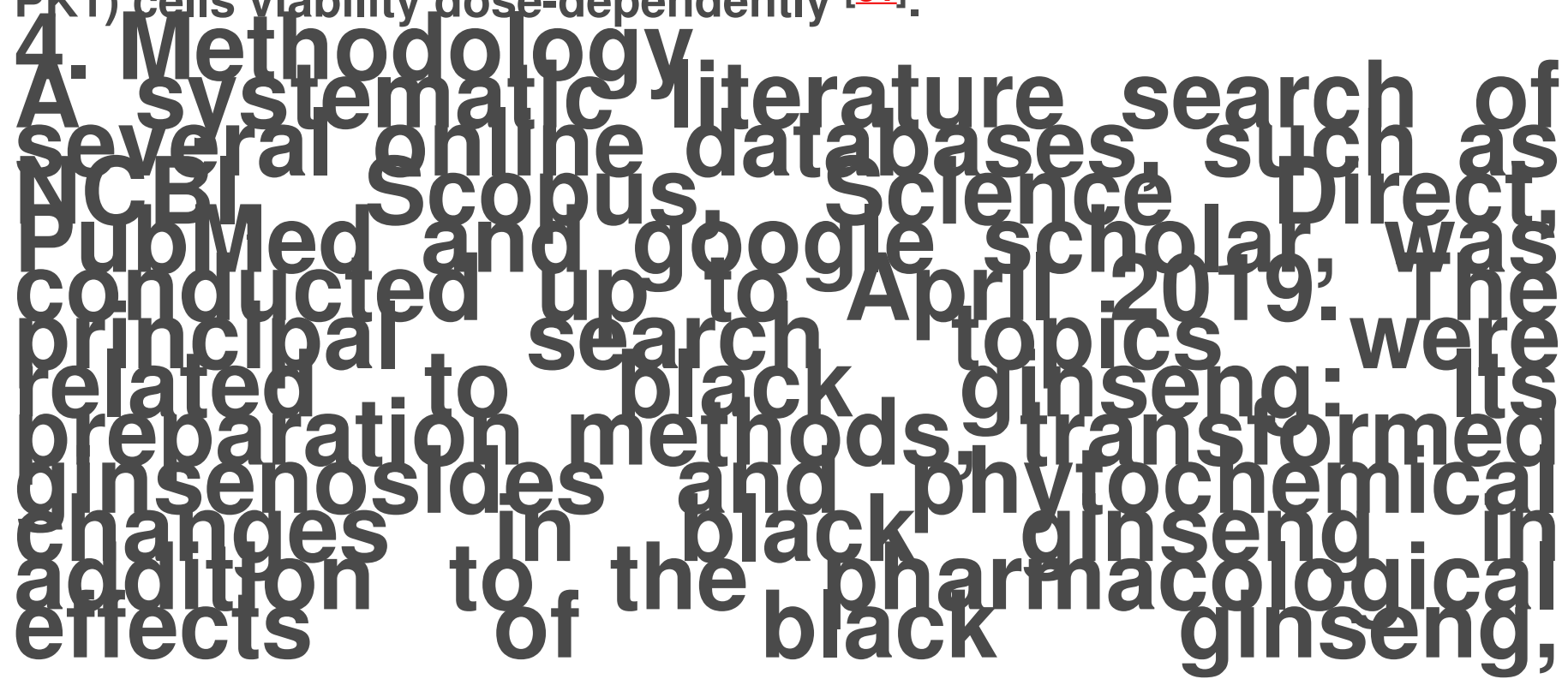




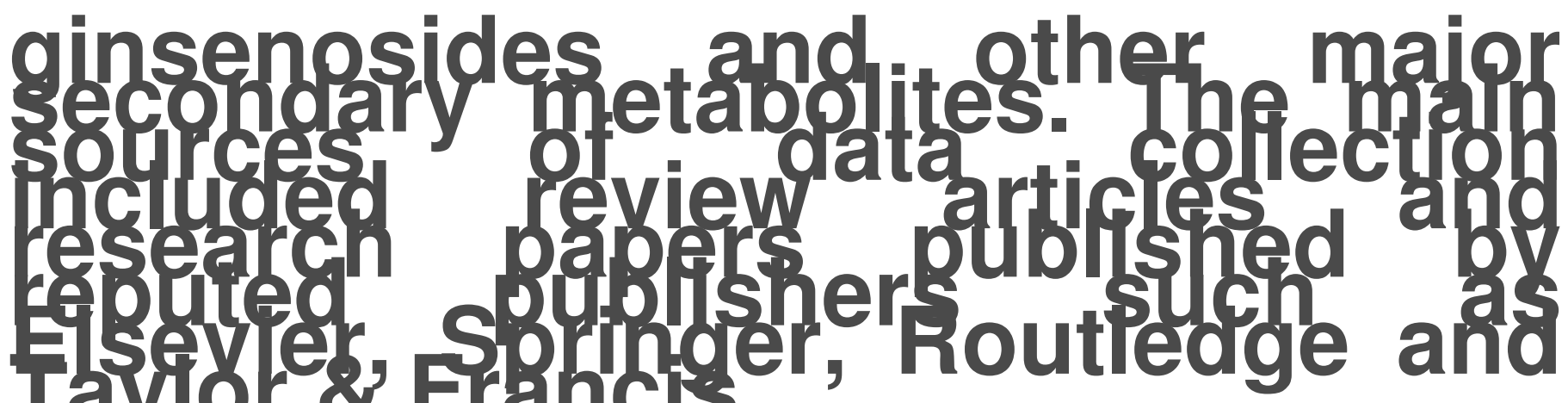

Tayo \& rancis.

Prospects

Ginseng was first discovereg and Janitionaly gnly ghite ofinseng

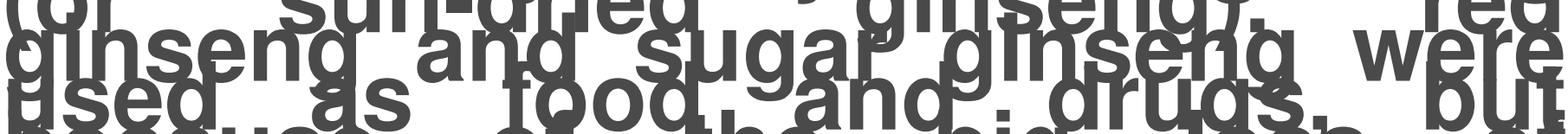
\% gnsengsides in suga ow bur resseg gis eno oro uit on storger secongry metabo tes onspe cr drogose to peers: nocseach ofinseng shar hewly

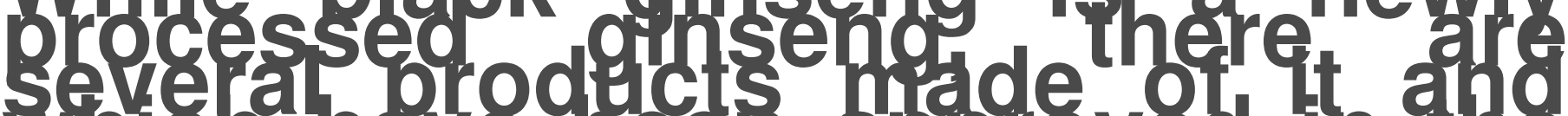
H hu rerth chlo on ares cofsugon

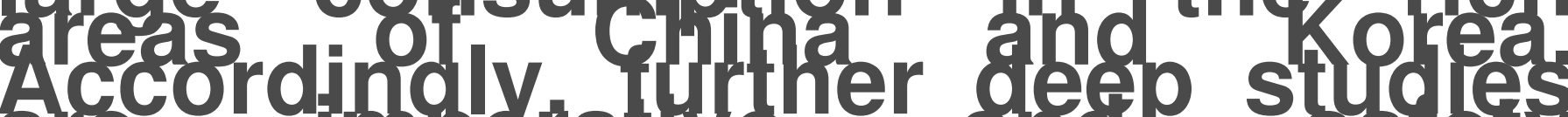
Acordingly ar ơs cond ${ }^{2}$ as a - 10 w axm $m$ m 8 the strond anticancer act $y$

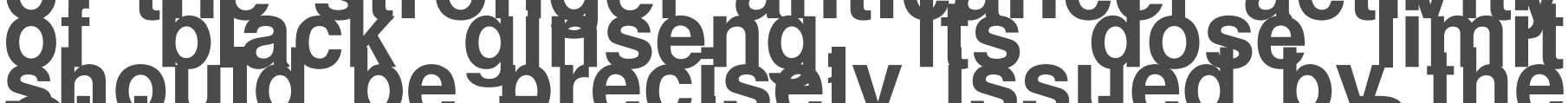
h०. Adnhistration (CFD'A) in Ching tointhateint alm the

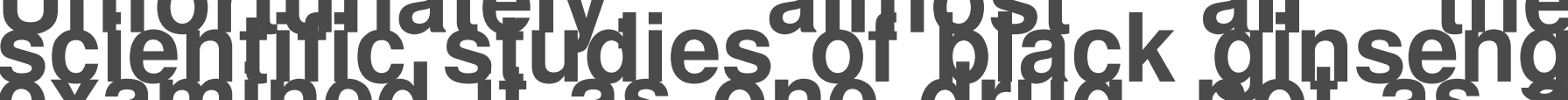
examined as one drug, irot as a 


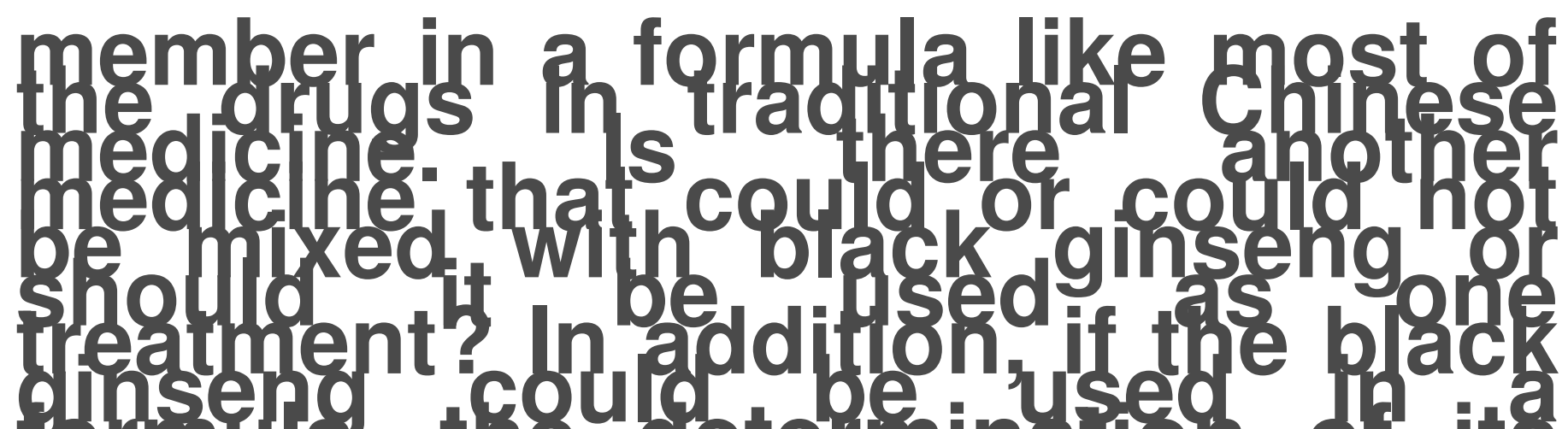

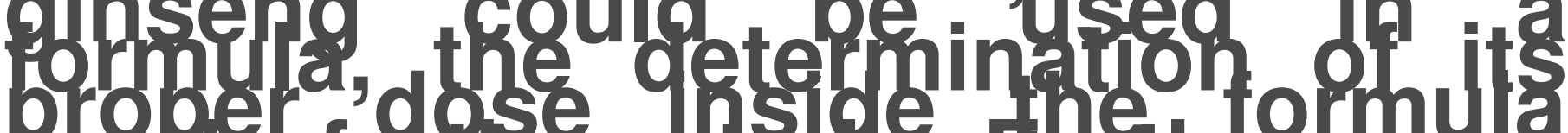
0)

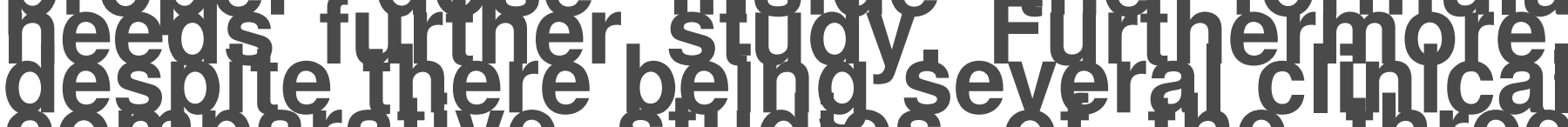

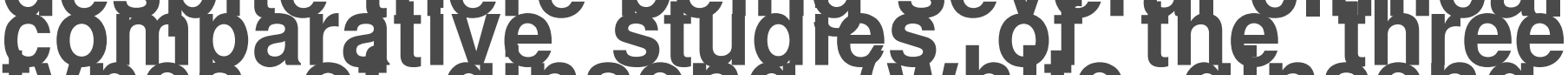

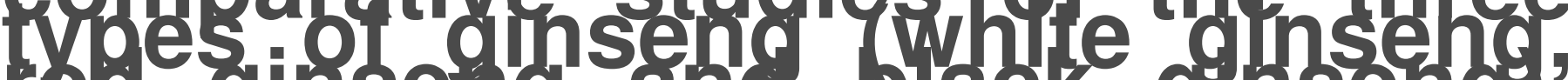

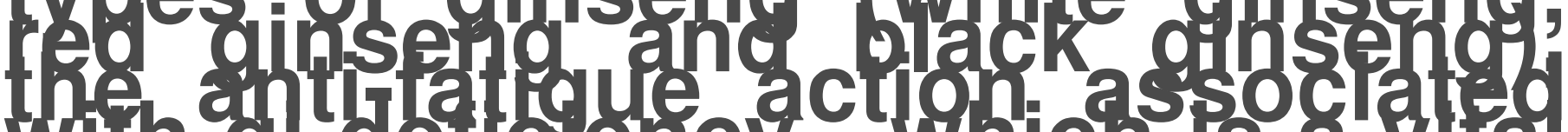
Mila 0 o

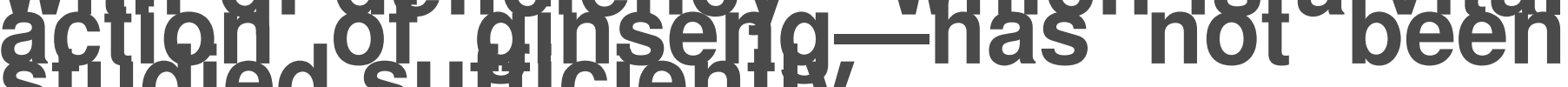

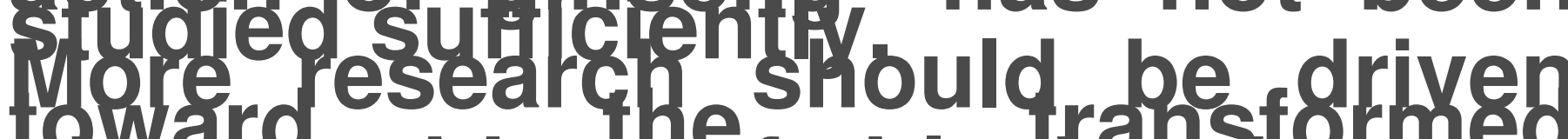
ous gns nsids of hlack sides sen bee getecté sén 月h

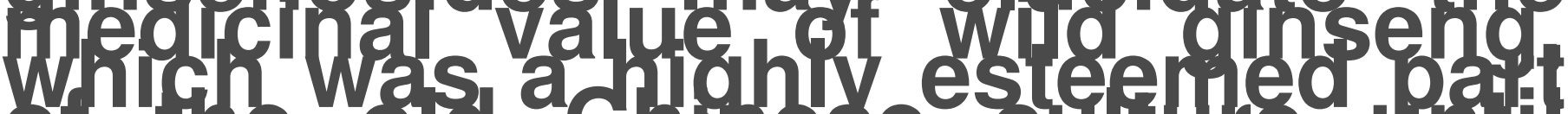
ot the old the se cut Bow A thition him gave tracks rmed the mor 3. jesently the ginseng de

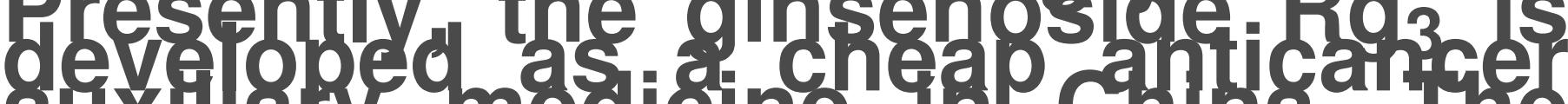
ax sta - nseng reresea he prepar ost vich g les a god a ce

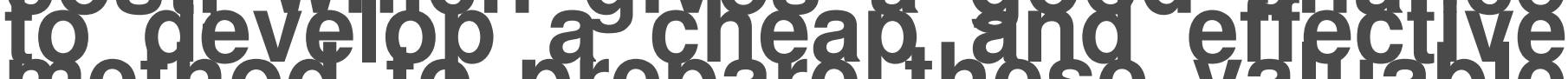
nethog prearie these valuable transiormed gihsenosides. 


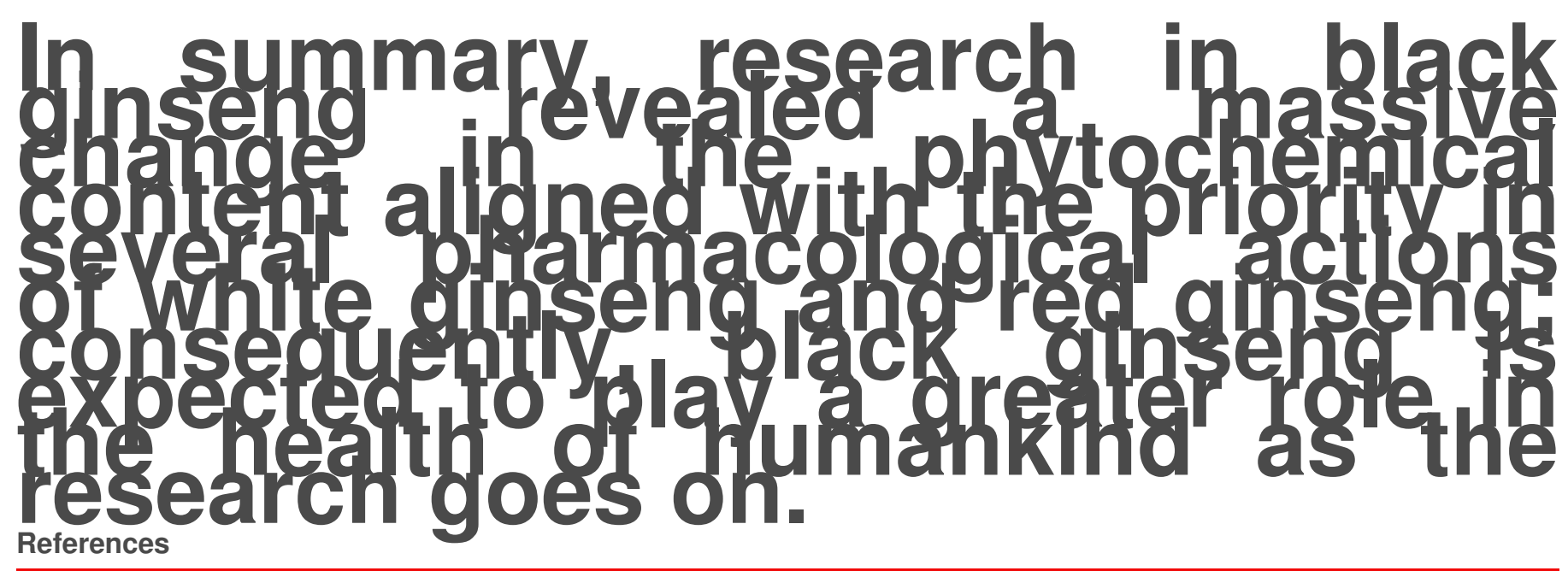

1. Yun, T.K. Brief introduction of Panax ginseng CA Meyer. J. Korean Med. Sci. 2001, 16, S3.

2. Nocerino, E.; Amato, M.; Izzo, A.A. The aphrodisiac and adaptogenic properties of ginseng. Fitoterapia 2000,71, S1-S5

3. Kang, S.; Min, H. Ginseng, the'immunity boost': The effects of Panax ginseng on immune system. J. GinsengRes. 2012, 36, 354.

4. Shin, H.R.; Kim, J.Y.; Yun, T.K.; Morgan, G.; Vainio, H. The cancer-preventive potential of Panax ginseng: Areview of human and experimental evidence. Cancer Causes Control. 2000, 11, 565-576.

5. Lee, C.H.; Kim, J.-H. A review on the medicinal potentials of ginseng and ginsenosides on cardiovasculardiseases. J. Ginseng Res. 2014, 38, 161-166.

6. Kim, H.J.; Kim, P.; Shin, C.Y. A comprehensive review of the therapeutic and pharmacological effects ofginseng and ginsenosides in central nervous system. J. Ginseng Res. 2013, 37, 8.

7. Matsuura, H.; Kasai, R.; TANAKA, O.; SARUWATARI, Y.; KUNIHIRO, K.; FUWA, T. Further studies ondammarane-saponins of ginseng roots. Chem. Pharm. Bull. 1984, 32, 1188-1192.

8. Nag, S.; Qin, J.; Wang, W.; Wang, M.-H.; Wang, H.; Zhang, R. Ginsenosides as anticancer agents: In vitro andin vivo activities, structure-activity relationships, and molecular mechanisms of action. Front. Pharmacol.2012, 3.

9. Christensen, L.P. Ginsenosides: Chemistry, biosynthesis, analysis, and potential health effects. Adv. FoodNutr. Res. 2008, 55, 1-99

10. Dou, D.-Q.; Chen, Y.-J.; Liang, L.-H.; PANG, F.-G.; Shimizu, N.; Takeda, T. Six new dammarane-type triterpenesaponins from the leaves of Panax ginseng. Chem. Pharm. Bull. 2001, 49, 442-446.

11. Dou, D.-Q.; Hou, W.-B.; Chen, Y.-J. Studies of the characteristic constituents of Chinese ginseng and Americanginseng. Planta Med. 1998, 64, 585-586

12. Fuzzati, N. Analysis methods of ginsenosides. J. Chromatogr. B 2004, 812, 119-133.

13. Chung, A.-S.; Park, K.M. Anticancer and Antineurodegenerative effects of Ginsenosides. In Studies in NaturalProducts Chemistry; Elsevier: Pakistan, 2016; Volume 50, pp. 131-158.

14. Shi, W.; Wang, Y.; Li, J.; Zhang, H.; Ding, L. Investigation of ginsenosides in different parts and ages of Panaxginseng. Food Chem. 2007, 102, 664-668.

15. Nam, K.-Y.; Lee, N.-R.; Moon, B.-D.; Song, G.-Y.; Shin, H.-S.; Choi, J.-E. Changes of ginsenosides and colorfrom black ginsengs prepared by steaming-drying cycles. Korean J. Med. Crop. Sci. 2012, 20, 27-35.

16. Jin, Y.; Kim, Y.-J.; Jeon, J.-N.; Wang, C.; Min, J.-W.; Noh, H.-Y.; Yang, D.-C. Effect of white, red and blackginseng on physicochemical properties and ginsenosides. Plant. Foods Hum. Nutr. 2015, 70, 141-145.

17. Sun, B.-S.; Pan, F.-Y.; Sung, C.-K. Repetitious steaming-induced chemical transformations and global qualityof black ginseng derived from Panax ginseng by HPLC-ESI-MS/MS n based chemical profiling approach.Biotechnol. Bioprocess. Eng. 2011, 16, 956.

18. Kang, K.S.; Kim, H.Y.; Pyo, J.S.; Yokozawa, T. Increase in the free radical scavenging activity of ginseng byheat-processing. Biol. Pharm. Bull. 2006, 29, 750-754.

19. Ellis, G. The maillard reaction. In Advances in Carbohydrate Chemistry; Elsevier, 1959; Volume 14, pp. 63-134.

20. Lee, Y.-S.; Im, D.-H.; Yang, J.-C.; Noh, D.-S.; Kim, K.-I.; Oh, S.-K.; Choi, K.-C.; Cha, Y.-H. Study on thequalitative discrimination of white, red, and black ginseng extract. Korean J. Food Nutr. 2011, 24, 138-143.

21. Cho, E.J.; Piao, X.L.; Jang, M.H.; Baek, S.H.; Kim, H.Y.; Kang, K.S.; Kwon, S.W.; Park, J.H. The eect ofsteaming on the free amino acid contents and antioxidant activity of Panax ginseng. Food Chem. 2008, 107,876-882.

22. Guo, N.; Zhu, L.; Song, J.; Dou, D. A new simple and fast approach to analyze chemical composition onwhite, red, and black ginseng. Ind. Crop. Prod. 2019, 134, 185-194.

23. IARCWorking Group on the Evaluation of Carcinogenic Risks to Humans; International Agency for Researchon Cancer; World Health Organization. Smokeless Tobacco and Some Tobacco-Specific N-nitrosamines; WorldHealth Organization: Geneva, Switzerland, 2007; Volume 89.

24. Kim, E.-K.; Lee, J.-H.; Cho, S.-H.; Shen, G.-N.; Jin, L.-G.; Myung, C.-S.; Oh, H.-J.; Kim, D.-H.; Yun, J.-D.;Roh, S.-S. Preparation of black panax ginseng by new methods and its antitumor activity. Korea J. Herbol.2008, 23, 85-92.

25. Song, G.Y.; Chung, K.J.; Shin, Y.J.; Lee, G.W.; Lee, S.Y.; Seo, Y.B. Study on Antiangiogenic Eect of BlackGinseng Radix. Kor. J. 
Herbol. 2011, 26, 83-90.

26. Chen, G.; Li, H.; Gao, Y.; Zhang, L.; Zhao, Y. Flavored black ginseng exhibited antitumor activity viaimproving immune function and inducing apoptosis. Food Funct. 2017, 8, 1880-1889.

27. Kim, A.-J.; Kang, S.-J.; Lee, K.-H.; Lee, M.; Ha, S.-D.; Cha, Y.-S.; Kim, S.Y. The chemopreventive potential andanti-inflammatory activities of Korean black ginseng in colon26-M3. 1 carcinoma cells and macrophages. J.Korean Soc. Appl. Biol. Chem. 2010, 53, 101105.

28. Kang, S.-J.; Han, J.-S.; Kim, A.-J. Ameliorate Eect of Black Ginseng on HepG2 Cell transplanted in BALB/cNude Mice. Korean J. Food Nutr. 2015, 28, 241-246.

29. Kim, S.-J.; Kim, A.K. Anti-breast cancer activity of Fine Black ginseng (Panax ginseng Meyer) and ginsenosideRg5. J. Ginseng Res. 2015, 39, 125-134.

30. Lee, Y.Y.; Saba, E.; Irfan, M.; Kim, M.; Yi-Le Chan, J.; Jeon, B.S.; Choi, S.K.; Rhee, M.H. The anti-inflammatoryand anti-nociceptive eects of Korean black ginseng. Phytomedicine 2019, 54, 169-181.

31. Han, M.-S.; Han, I.-H.; Lee, D.; An, J.M.; Kim, S.-N.; Shin, M.-S.; Yamabe, N.; Hwang, G.S.; Yoo, H.H.;Choi, S.-J. Beneficial eects of fermented black ginseng and its ginsenoside 20 (S)-Rg3 against cisplatin-inducednephrotoxicity in LLC-PK1 cells. J. Ginseng Res. 2016, 40, 135-140.

32. Shin, Y.J.; Jang, H.H.; Song, G.Y. Study on Anti-atopic Eects of Black Ginseng. Kor. J. Aesthet. Cosmetol 2012,10, 91-97.

33. Kang, J.A.; Song, H.; Byun, E.; Ahn, N.; Kim, H.; Nam, Y.R.; Lee, G.H.; Jang, B.; Choi, D.S.; Lee, D.Gamma-irradiated black ginseng extract inhibits mast cell degranulation and suppresses atopic dermatitis-likeskin lesions in mice. Food Chem. Toxicol. 2018, 111, 133143.

34. Hu, J.-N.; Liu, Z.; Wang, Z.; Li, X.-D.; Zhang, L.-X.; Li, W.; Wang, Y.-P. Ameliorative eects and possiblemolecular mechanism of action of black ginseng (Panax ginseng) on acetaminophen-mediated liver injury.Molecules 2017, 22, 664.

35. Kang, O.-H.; Shon, M.-Y.; Kong, R.; Seo, Y.-S.; Zhou, T.; Kim, D.-Y.; Kim, Y.-S.; Kwon, D.-Y. Anti-diabeticeect of black ginseng extract by augmentation of AMPK protein activity and upregulation of GLUT2 andGLUT4 expression in db/db mice. Bmc Complementary Altern. Med. 2017, 17, 341.

36. Bak, M.; Jeong, W.; Kim, K. Detoxifying eect of fermented black ginseng on H2O2-induced oxidative stressin HepG2 cells. Int. J. Mol. Med. 2014, 34, 1516-1522.

37. Kim, J.H.; Pan, J.H.; Cho, H.T.; Kim, Y.J. Black ginseng extract counteracts streptozotocin-induced diabetes inmice. PLoS ONE 2016, 11, e0146843.

38. Kim, S.-N.; Kang, S.-J. Eects of black ginseng (9 times-steaming ginseng) on hypoglycemic action andchanges in the composition of ginsenosides on the steaming process. Korean J. Food Sci. Technol. 2009, 41,77-81.

39. Seo, Y.-S.; Shon, M.-Y.; Kong, R.; Kang, O.-H.; Zhou, T.; Kim, D.-Y.; Kwon, D.-Y. Black ginseng extract exertsanti-hyperglycemic eect via modulation of glucose metabolism in liver and muscle. J. Ethnopharmacol. 2016,190, 231-240.

40. Lee, M.R.; Kim, B.C.; Kim, R.; Oh, H.I.; Kim, H.K.; Choi, K.J.; Sung, C.K. Anti-obesity eects of black ginsengextract in high fat diet-fed mice. J. Ginseng Res. 2013, 37, 308.

41. Saba, E.; Jeon, B.R.; Jeong, D.-H.; Lee, K.; Goo, Y.-K.; Kim, S.-H.; Sung, C.-K.; Roh, S.-S.; Kim, S.D.; Kim, H.-K.Black ginseng extract ameliorates hypercholesterolemia in rats. J. Ginseng Res. 2016, 40, 160-168.

42. Kim, A.-J.; Yoo, H.-S.; Kang, S.-J. Ameliorative eect of black ginseng on diabetic complications inC57BLKS/J-db/db mice. Korean J. Food Nutr. 2012, 25, 99-104.

43. Park, H.-J.; Kim, A.-J.; Cheon, Y.-P.; Lee, M. Anti-obesity eects of water and ethanol extracts of black ginseng.J. Korean Soc. Food Sci. Nutr. 2015, 44, 314-323.

44. Lee, M.-R.; Yun, B.-S.; Sun, B.-S.; Liu, L.; Zhang, D.-L.; Wang, C.-Y.; Wang, Z.; Ly, S.-Y.; Mo, E.-K.; Sung, C.-K.Change of ginsenoside $\mathrm{Rg} 3$ and acetylcholinesterase inhibition of black ginseng manufactured by grapejuice soaking. J. Ginseng Res. $2009,33,349-354$.

45. Lee, M.R.; Yun, B.S.; Sung, C.K. Comparative study of white and steamed black Panax ginseng, P.quinquefolium, and P. notoginseng on cholinesterase inhibitory and antioxidative activity. J. GinsengRes. 2012, 36, 93.

46. Lee, M.-R.; Yun, B.-S.; Liu, L.; Zhang, D.-L.;Wang, Z.;Wang, C.-L.; Gu, L.-J.;Wang, C.-Y.; Mo, E.-K.; Sung, C.-k.Effect of black ginseng on memory improvement in the amnesic mice induced by scopolamine. J. GinsengRes. 2010, 34, 51-58.

47. Saba, E.; Jeong, D.-H.; Roh, S.-S.; Kim, S.-H.; Kim, S.-D.; Kim, H.-K.; Rhee, M.-H. Black ginseng-enrichedChong-Myung-Tang extracts improve spatial learning behavior in rats and elicit anti-inflammatory eectsin vitro. J. Ginseng Res. 2017, 41, 151-158.

48. Park, H.-J.; Shim, H.S.; Kim, K.S.; Shim, I. The protective eect of black ginseng against transient focalischemia-induced neuronal damage in rats. Korean J. Physiol. Pharmacol. 2011, 15, 333-338.

49. Lee, M.R.; Begum, S.; Sung, C.K. Eect of red and black ginseng on cholinergic markers, presynaptic markers, and neurotrophins in the brain of aged mice. Food Sci. Biotechnol. 2017, 26, 1743-1747.

50. Oh, C.-H.; Kim, G.-N.; Lee, S.-H.; Lee, J.-S.; Jang, H.-D. Eects of heat processing time on total phenoliccontent and antioxidant capacity of ginseng Jung Kwa. J. Ginseng Res. 2010, 34, 198-204.

51. Kim, H.-J.; Lee, J.-Y.; You, B.-R.; Kim, H.-R.; Choi, J.-E.; Nam, K.-Y.; Moon, B.-D.; Kim, M.-R. Antioxidantactivities of ethanol extracts from black ginseng prepared by steaming-drying cycles. J. Korean Soc. Food Sci.Nutr. 2011, 40, 156-162.

52. Lee, S.-R.; Kim, M.-R.; Yon, J.-M.; Baek, I.-J.; Park, C.G.; Lee, B.J.; Yun, Y.W.; Nam, S.-Y. Black ginseng inhibitsethanol-induced teratogenesis in cultured mouse embryos through its eects on antioxidant activity. Toxicol.Vitro 2009, 23, 47-52.

53. Saba, E.; Lee, Y.Y.; Kim, M.; Kim, S.-H.; Hong, S.-B.; Rhee, M.H. A comparative study on immune-stimulatoryand antioxidant activities 
of various types of ginseng extracts in murine and rodent models. J. Ginseng Res.2018, 42, 577-584.

54. Gong, L.-H.; Lei, T.; Zhang, Z.-L.; Liang, Q.-C.; Zhai, F.-G.; Wu, Y.-Y.; Zhang, X.-P.; Liu, J.-Q.; Liu, J.-W.Purification, compositional analysis and antioxidant properties of polysaccharides from black ginseng. Trop.J. Pharm. Res. 2018, 17, 1317-1324.

55. Jo, G.S.; Chai, H.-Y.; Ji, H.J.; Kang, M.H.; Kang, S.-J.; Ji, J.-G.; Kim, D.J.; Lee, B.J. Enhancement of exercisecapacity by black ginseng extract in rats. Lab. Anim. Res. 2010, 26, 279-286.

56. Lee, S.-Y.; Go, G.-Y.; Vuong, T.A.; Kim, J.W.; Lee, S.; Jo, A.; An, J.M.; Kim, S.-N.; Seo, D.-W.; Kim, J.-S. Blackginseng activates Akt signaling, thereby enhancing myoblast dierentiation and myotube growth. J. GinsengRes. 2018, 42, 116-121.

57. Kim, M.; Choi, S.Y.; Kim, S.S.; Kim, J.; Boo, S.J.; Hur, J. Function of Korean black ginseng: Improvement ofandropause symptoms by a complex extract of black ginseng and fenugreek in TM3 Leydig cells and agedrats. J. Ethn. Foods 2016, 3, 228-234.

58. Wang, J.; Li, S.; Fan, Y.; Chen, Y.; Liu, D.; Cheng, H.; Gao, X.; Zhou, Y. Anti-fatigue activity of the water-solublepolysaccharides isolated from Panax ginseng CA Meyer. J. Ethnopharmacol. 2010, 130, 421-423.

59. Pham, Q.L.; Jang, H.J.; Kim, K.B. Anti-wrinkle eect of fermented black ginseng on human fibroblasts. Int. J.Mol. Med. 2017, 39, 681686.

60. Park, J.Y.; Lee, D.S.; Kim, C.; Shin, M.; Seo, C.; Shin, H.; Hwang, G.S.; An, J.M.; Kim, S.; Kang, K.S. Eects offermented black ginseng on wound healing mediated by angiogenesis through the mitogen-activated proteinkinase pathway in human umbilical vein endothelial cells. J. Ginseng Res. 2017, 42, 524-531.

61. Lee, M.; Oh, C.; Li, Z.; Li, J.; Wang, C.; Wang, Z.; Gu, L.; Lee, S.; Lee, J.; Lim, B. Evaluation of the Oral AcuteToxicity of Black Ginseng in Rats. J. Ginseng Res. 2011, 35, 39-44.

62. Zhang, Y.H.; Li, H.D.; Li, B.; Jiang, S.D.; Jiang, L.S. Ginsenoside Rg3 induces DNA damage in humanosteosarcoma cells and reduces MNNG-induced DNA damage and apoptosis in normal human cells. Oncol.Rep. 2014, 31, 919-925.

63. Jung, K.; An, J.M.; Eom, D.; Kang, K.S.; Kim, S. Preventive eect of fermented black ginseng againstcisplatin-induced nephrotoxicity in rats. J. Ginseng Res. 2017, 41, 188-194.

64. Jeong, H.; Lim, C.; Cha, B.; Choi, S.; Kwon, K. Component analysis of cultivated ginseng, cultivatedwild ginseng, and wild ginseng and the change of ginsenoside components in the process of red ginseng.J. Pharmacopunct. 2010, 13, 63-77.

65. Nag, S.; Qin, J.; Wang, W.; Wang, M.-H.; Wang, H.; Zhang, R. Ginsenosides as anticancer agents: In vitro andin vivo activities, structure-activity relationships, and molecular mechanisms of action. Front. Pharmacol.2012, 3.

66. Nag, S.; Qin, J.; Wang, W.; Wang, M.-H.; Wang, H.; Zhang, R. Ginsenosides as anticancer agents: In vitro andin vivo activities, structure-activity relationships, and molecular mechanisms of action. Front. Pharmacol.2012, 3.

\section{Keywords}

Black ginseng; Transformed ginsenosides; Anti-cancer; Anti-inflammatory; Ginsenoside-Rg3; Ginsenoside-Rg5

(C) 2019 by the author(s). Distribute under aCreative Commans CC BY license 